\title{
El nombre en los procesos judiciales de cambio, supresión 0 adición de nombre en los juzgados civiles o mixtos de Lima Metropolitana en el Perú 2010-2013
}

\section{The name change court proceedings, deletion or addition name in the civil courts or mixed in Lima Peru: 2010-2013}

\author{
Recibido: 17/07/2015 Revisado: 11/08/2015 Aceptado: 03/09/2015
}

Jesús Yuseff Sotomayor Rivera ${ }^{1}$

\begin{abstract}
RESUMEN
Objetivo: Analizar los factores jurídicos que determinan el nombre en los procesos judiciales de cambio, supresión o adición de nombre en los juzgados civiles o mixtos de Lima Metropolitana en el Perú: 2010-2013. Materiales y métodos: Se realizó un análisis a las sentencias judiciales referentes al cambio, supresión o adición de nombre que fueron emitidas por los juzgados civiles o mixtos de Lima Metropolitana en el Perú durante los años 2010 al 2013. Los métodos empleados son: No experimental porque no se manipuló variables ya que observamos los hechos tal y como se han dado en la realidad. Ex - Post facto ya que estudiamos los hechos ya ocurridos. Transversal porque la recolección de datos se realizó en un solo momento y tiempo único. Resultados: A consecuencia de los vacíos legales, es decir, nuestro Código Civil no contempla un límite para los prenombres, en su calidad ni en su cantidad al colocar el nombre a un menor de edad. Conclusiones: El Derecho y la Ley, no pueden mantenerse impasibles frente al hecho que existan padres que de modo irreflexivo e irresponsable decidan ponerles a sus hijos prenombres arbitrarios, claramente lesivos a su dignidad y a sus posibilidades de un desarrollo sin mortificaciones ni inconvenientes.
\end{abstract}

Palabras clave: El nombre, el prenombre, el apellido, la identidad, la dignidad.

\section{ABSTRACT}

Objective: To analyze the cognitive, political, regulatory and economic factors that determine the name in the judicial processes of change, deletion and addition of name in the civil of Lima metropolitan in Peru: 2010-2013. Materials and methods: It was made an analysis to those judicial sentences related to change, deletion, or addition of name that were issued for the civil of Lima metropolitan in Peru during years 2010 to 2013. The methods employed are Not experimental because it will not manipulate variables doubt to we will observe facts as they are occurring in reality. Ex Post Facto because we studying facts already occurred. Cross because data collection was made in only a moment and in a unique time. Results: As a result of loopholes, in other words, our Civil Code does not provide a limit for prenames, in its quality or quantity when putting a name to a minor. Conclusions: The rights and the law cannot remain impassive faced with the fact that there are parents who decide in a thoughtless and irresponsible way to put their children arbitrary prenames, clearly harmful to their dignity and their possibility to grow without mortification or inconvenience.

Keywords: The name, prename, last name, identity, dignity. INTRODUCCIÓN

La protección jurídica del nombre no puede encararse como la mera defensa de un derecho subjetivo, el ataque al nombre mediante la asignación de prenombres ridículos extravagantes e indecoros es un ataque a su personalidad. El derecho al nombre está tutelado por un derecho macro que es el de la identidad. A la vez que derecho, el nombre es un deber (Código Civil, 1984). El nombre es inmutable, inalterable, inmodificable. Impuesto y debe llevarse como tal. La eventual modificación del nombre genera confusión e impedirá identificar a la persona, desvirtuando la función individualizadora propia de esta institución (Vásquez, 1997). La persona tiene el deber de mantener su nominación. En el Perú, hoy en día el cambio, la supresión o la adición de nombre en los juzgados especializados se ha convertido en un tema tedioso para la sociedad y para quienes tienen que dar solución respecto a esta problemática. Los prenombres que en su conjunto es llamado nombre (prenombres y apellidos) se han convertido en un juego donde la exageración, la búsqueda del protagonismo y donde la idea errónea de marcar la diferencia dentro del grupo social ha conllevado a que los padres designaran prenombres ofensivos, obscenos, extravagantes e inclusive indecorosos para sus menores hijos.

Entre los factores que se pudo describir a la cual obedece la situación actual; es la exigua capacidad cognoscitiva de los progenitores en el momento de asignar los prenombres a los hijos recién nacidos. Como se sabe el prenombre es el elemento individual característico del sujeto libre de toda vinculación preestablecida, pues la palabra con la que viene constituida este signo es elegida libre y arbitrariamente por quien tiene la facultad de imponerlo al recién nacido (Vega, 1995). Muchas veces la idiosincrasia (dependiendo nivel socioeconómico y cultural) que cada ciudadano posee, hace que decidan por prenombres que al corto y largo plazo conlleven a sus hijos a las burlas, al maltrato psicológico y al maltrato físico. Otro factor importante a mencionar son los vacíos legales, es decir, nuestro Código Civil no contempla un límite para los prenombres, en su calidad ni en su cantidad al colocar el nombre a un menor de edad. Esto muchas veces perjudica directamente a los receptores de nombres indecorosos debido a que ata de manos a los registradores del Reniec 
ya que no poseen ninguna autoridad ni tampoco tienen argumentos legales para decir que no es correcto el nombre que los padres mencionan al momento de la inscripción del acta de nacimiento. Por lo tanto, este vacío legal respecto al control de la forma y uso del prenombre muestra una considerable cantidad de personas que no se encuentran satisfechas con el prenombre que les ha sido asignado y que por múltiples razones se ven obligados(as) a solicitar el cambio, supresión o adición de nombre en los juzgados civiles o mixtos.

Es importante mencionar que el Estado mediante las leyes tiene escasa participación y actúa de manera pasiva ante los vacíos legales; es decir, existe una falta de voluntad política para poder mejorar esta situación donde los niños y adolescentes son propensos a llevar consigo prenombres arbitrarios y claramente lesivos a su identidad. Es claro la indiferencia del Estado ante esta realidad innegable de la asignación de prenombres inadecuados de parte de los padres para con sus menores hijos; ni las entidades en salvaguarda del Interés Superior del Niño como el Ministerio de la Mujer y Poblaciones Vulnerables, Ministerio de Justicia y Derechos Humanos, Ministerio Público, Demunas u otros que actúan para cambiar y mejorar esta situación. Entonces se concluye que nos encontramos inmersos en un gran problema social. Y por lo tanto somos expuestos hacer objetos de burla desde antes del nacimiento, porque nuestros progenitores lamentablemente disponen con autoridad y libertad absoluta al no existir impedimento en la designación del prenombre desde antes que naciéramos. Esto favorece a que incrementen los índices de maltratos en los hogares, colegios y también en el ámbito laboral.

Dentro del corto plazo son los niños y jóvenes quienes se perjudican en la mayoría de los casos en colegios de cualquier nivel (inicial, primaria y secundaria) con los insultos, burlas y que terminan siendo proclives al bullying infantil. El resultado es el aislamiento de los niños dentro de la sociedad, los suicidios por ser tratado como objeto y no como persona y finalmente terminan siendo violentados por sus transgresores en edad escolar muchas veces no se dan cuenta que actúan erróneamente afectando lesivamente la personalidad del niño o adolescente, máxime que la personalidad se forma hasta los 6 años. A largo plazo somos nosotros los adultos quienes también vivimos frecuentemente el bullying laboral, pero aquí sí hay que detenernos, porque hay una marcada diferencia entre los niños, adolescentes y adultos siendo estos últimos capaces de distinguir lo correcto de lo incorrecto pero sin embargo no lo discernimos con las acciones. En un escenario pesimista debido al problema de que los padres nos asignan prenombres inadecuados, muchos de nuestros compañeros nos maltratan psicológicamente pero nos defendemos; es decir, hay una confrontación que se determinará por el uso de la fuerza, en pocas palabras la sociedad convierte en personas violentas a quienes por poder buscar defenderse o imponer respeto hacen uso de una legítima defensa.

La otra parte también afectada por estos vacíos legales son los juzgados civiles o mixtos que se ven llenos de demandas, trámites engorrosos y carga laboral, por dar solución pronta a las necesidades de índole social para que las personas afectadas sean respetadas(os) en su máxima expresión, el cual es la dignidad. Los escenarios presentados y descritos en los párrafos anteriores nos permiten llegar a una conclusión, donde todos serán afectados de manera negativa como resultado de esta problemática; tanto para las personas quienes poseen los prenombres vejatorios y para las instituciones que apoyan en buscar y llegar a una solución pronta y efectiva. Sin embargo; hay alternativas de solución para que esto mejore y cambie en pocos años. Empezaremos por el Congreso de la República, ya que son ellos quienes tienen la potestad de modificar las leyes, proponiendo un proyecto de ley para modificar el artículo 19 de Código Civil, acorde del artículo 2 inciso 1 de la Constitución Política, donde se menciona expresamente que toda persona tiene derecho a la identidad y a su integridad moral y psíquica (...). Con ello se busca que los padres quienes son los responsables de asignar los prenombres de los recién nacidos con la modificatoria del artículo en mención primara la calidad en la asignación y una limitación en cantidad de prenombres, además dichos prenombres deben ser diferentes en su estructura de composición para que no exista confusión con los apellidos, teniendo en cuenta también el género de quien lo porta. La otra alternativa también es importante mencionar que es un cambio en nuestra educación, donde lo primordial es inculcar valores y principios a los niños y con ello esperar que sean buenos ciudadanos y que parte de su formación sea el respeto por los demás he aquí el primer valor y fuente de desarrollo personal que los convierta en conductores del cambio en la sociedad.

Además una concientización de los problemas de maltratos psicológicos y físicos nos permite darnos cuenta que ya es hora de cambiar nuestros patrones de conducta inadecuadas. Es importante tener claro las consecuencias de las actitudes inapropiadas son las manifestaciones discriminatorias ante personas sujetas a prenombres denigrantes.

Con las modificaciones respectivas al nombre en nuestra norma sustantiva; permitirá que los juzgados (jueces y auxiliares jurisdiccionales) tengan un desempeño más eficiente y eficaz para otros trámites que si merecen la importancia de su participación y no desgastar tiempo. Estas situaciones difíciles de enfrentar cuando se forma la personalidad de un menor, genera traumas infantiles que no sólo provocan problemas psicológicos que repercuten en la edad adulta. Los niños son extremadamente vulnerables a los acontecimientos traumáticos y muchos acontecimientos que los adultos consideran ordinarios, ellos los experimentan como amenazantes para su vida como dice Juan Espinoza Espinoza citando a Tommasini: "El hombre en su dimensión de coexistencia tiene derecho a que no se deforme, distorsione o desnaturalice su propia personalidad" (Gaceta Jurídica, 2007).

\section{Origen del cambio, supresión o adición del nombre en el Perú}

El artículo 33 del Reglamento de Inscripción del Registro Nacional de Identificación y Estado Civil, Decreto Supremo No 015-98 PCM (publicado el 25/4/98) llenó un gran vacío en la legislación peruana, sobre el agudo problema de la asignación de nombres de pila o prenombres en beneficio de los niños y adolescentes; sin embargo, fue derogado 72 horas después por el Decreto Supremo $N^{\circ} 016-98$ PCM, sin haberse generado un debate sobre el tema. Aunque es verdad que la norma era imperfecta, podía mejorarse la redacción del artículo a través de modificaciones adecuadas, y creando a su vez los mecanismos legales que permitieran su aplicación. Este tema fue arduamente debatido en el seno del Seminario Nacional de Registradores Civiles, organizado por el Concejo Provincial de Trujillo del 27 al 29 de setiembre de 1996, puesto que en el Perú muchas veces se asignan a las personas nombres que no guardan relación con nuestra cultura y que son inadecuados para seres humanos, generando con ello un problema a los menores en su futuro proceso de socialización y en su vida de relación. 
Existen casos en que a un menor se le ha puesto el nombre de Rock and Roll, Made in Usa, Neurona $\mathrm{H}_{2} \mathrm{O}$, (Espinoza, 2004). También se tiene información proporcionada por el Reniec donde se muestra ejemplo claro de la exagerada libertar para designar prenombres en este caso por una celebración en especial por ejemplo por el Día del Abogado hay personas quienes llevan como prenombres los símbolos de esta profesión, tenemos personas que se llaman Ley, Proceso, Civil, entre otros. Incluso por el aniversario patrio también existen personas portadores que llevan en sus prenombres Perú y también hay quienes se llaman Patria.

Otro ejemplo claro es cuando se celebra el aniversario de alguna región de nuestro país por ejemplo en el aniversario de Cusco hay quienes se llaman Porca, Inti Raymi y la lista es larga; también según el Reniec hay quienes se llaman Corongo, Urpi, Amaru, Wara, etc, esto por el día de la diversidad cultural; finalmente hay quienes se identifican demasiado con sus laborales profesionales u oficios que llevan asignado sus prenombres como por ejemplo Estilista, Abogado etc. Son solo algunos ejemplos por citar. Estos nombres son evidentemente inadecuados para personas; sin embargo, por la libertad de los padres al respecto se comete este tipo de atrocidades y los registradores poco o nada pueden hacer porque la Ley no los faculta para oponerse o rechazar un nombre de esta naturaleza, como sí sucede en Argentina, por ejemplo, donde el registrador se opone, y si los padres no están de acuerdo, apelan la decisión del registrador a la Corte, quien es la que finalmente decide sobre la procedencia del nombre, pudiendo incluso cambiarlo. Sobre las facultades de oposición del registrador a nombres inadecuados; creemos que el registrador civil podría oponerse o negarse a inscribir un niño cuyo nombre es manifiestamente inadecuado para seres humanos, respaldándose en las disposiciones del Código del Niño y del Adolescente que expresan que se debe proporcionar al niño todas las condiciones para su desarrollo físico y psicológico, y un nombre inadecuado le va a causar indiscutiblemente problemas en sus relaciones sociales futuras; sentirá vergüenza, sufrirá probablemente mofas sobre su nombre y ello indudablemente afectará su proceso de socialización. Los prenombres deben ser acordes no denigrantes ni que causen hilaridad, como los siguientes cuya existencia se ha comprobado: Punto, Coma, Yahoo, Justicia, Hashtag, Lesbiana, Semáforo, Prostituta, Superman, Dos a Uno, Elvio Lado, Excremento, Débora Mel, Ambulancia, Paracetamol, Alama Marcela, Eleonor De Guerra, Osama Bin Laden, Zoila Maravillosa, Semen de Dioses, Estatua de La Libertad, Conflicto Internacional y Perfecto Ladrón Honrado (Varsi, 2014).

Por lo expuesto y para garantizar que los recién nacidos cuenten con un nombre que guarde relación con nuestro idioma y nuestra cultura, el Reglamento de Inscripciones del Reniec debe considerar una norma que evite nombres extravagantes, inadecuados, rimbombantes, cuya escritura dificultosa a veces genera errores de inscripción, constituyendo a veces sonidos de difícil pronunciación hasta para los propios padres. $Y$ es que la Ley no puede ser solo un instrumento regulador, sino también educador, que oriente nuestra idiosincrasia saturada de modelos y aptitudes alienadas, sin que esto signifique coactar o restringir la libertad de las personas (los padres) al respecto (Duran, 1997). El derecho moderno es promotor del desarrollo social y puede coadyuvar a la superación de ciertos esquemas de pensamiento caduco respecto de la asignación de prenombres a los hijos.
El Dr. Aníbal Torres Vásquez, cuando en su obra de Derecho Civil expresa que "es hora de contribuir a educar a nuestra gente, a enseñarle a valorarse a sí misma, a superar su complejo de inferioridad por creer en la superioridad de las razas, que tanto daño nos hace. Hay que enseñarles que por el hecho de poner un nombre extranjero a su hijo, este no se va a convertir en alemán, inglés, francés, etc., para que comprendan que un niño peruano no es ni un ápice menos que cualquier niño del mundo (Gaceta Jurídica, 2004)". Es necesario ahondar en este punto porque la asignación de nombres es un tema que debe estar adecuadamente legislado en nuestra sociedad.

El nombre es la expresión visible y social mediante el cual se identifica a la persona, por lo que adquiere singular importancia dentro de los derechos de la persona. Esta peculiar función hace que la facultad de la persona a ser reconocida por su propio nombre implique también el deber, frente a la sociedad, de no cambiar de nombre, salvo por motivos justificados y mediando autorización judicial. (Fernández, 2004).

El aporte del estudio del cambio, supresión o adición del nombre en el Perú

Conveniencia: Es importante porque existe un vacío legal que existe en el Código Civil y el reglamento del Reniec, no sólo en referencia a los prenombres inapropiados, tampoco existen límites al número de prenombres, dentro de esta perspectiva, resulta un gravísimo error cerrar los ojos ante la realidad y pretender que el derecho es algo estático e inamovible, alrededor del que giran las sociedades. Ciertamente tampoco se puede creer que se trata de lo contrario, es decir, que el derecho constituya el satélite de la sociedad y que por tanto debe amoldarse por completo a cualquier cambio que se produzca. No es una cosa ni la otra, Ambos, derecho y sociedad, deben encontrarse unidos por un vínculo de armonía mediante el cual el primero considere dentro de sus parámetros a la segunda, y ésta a su vez debe someterse a las pautas que el derecho le fije. Por lo tanto el objetivo es que la sociedad como conjunto sea justa y que los individuos que la conforman lo interioricen así y se sientan satisfechos de su sistema. En consecuencia el derecho está al servicio de estos fines, y por ello se mantiene en constante evolución, y siendo la codificación su vehículo de manifestación más idónea; es necesario la actualización o adecuación de la norma a la realidad.

Relevancia Social: El derecho al honor ataña a todos los ciudadanos, de forma pública siendo uno de los derechos fundamentales por su carácter personalísimo debiendo ser protegido frente a los excesos de la libertad que aún da el Código Civil. Y más aún por aportar a la sociedad a minimizar los traumas en los niños y jóvenes del país, y estén conformes con su prenombres, en la actualidad el fenómeno del bullying que se produce en los centros escolarizados debe ser un tema importante en las decisiones y nuevas estrategias por parte del sector educativo porque se puede determinar que, una de las causas de este rechazado fenómeno es el uso de nombres indecorosos que convierten a los niños en víctimas; lo cual llama la atención a la Ley y los padres a concientizar en este tema, llevando muchas veces al niño o adolescente a tener conductas depresivas, lo cual se manifiesta de diversas maneras lo más común en nuestra sociedad; es el aislamiento de los niños y adolescentes de su entorno produciendo con ello un daño en la identidad psicosomática del ser humano. 
Implicancias prácticas: Debido a que se adopta una postura de protección y defensa de los derechos humanos primordiales, que al parecer se han dejado de lado pues la Ley tiene escasa participación en sus artículos y no interviene directamente sino de forma pasiva, corrigiendo estos vacíos legales se ahorraría engorrosos trámites con el cambio de nombres (prenombres) a futuro.

Valor Teórico: El Código Civil de nuestro país en cuanto al prenombre no ha sido actualizado en comparación de países vecinos como Argentina, en los que la Ley esta terminante prohibido la asignación de prenombres inadecuados claramente lesivos a la dignidad de quien lo porta, el derecho civil peruano no contempla un límite para los prenombres en su calidad ni cantidad al colocar el nombre a un menor de edad. No existe propuesta en el Congreso de la República sobre el tema en mención, en la actualidad hay una propuesta legislativa que modifican varios artículos del Código Civil, pero no se contempla modificación alguna en lo concerniente al nombre en este caso al artículo $19^{\circ}$ del código civil ni hace alusión a su reglamento del Reniec.

Se debe tener en cuenta que no hay estudios anteriores serios sobre el tema de investigación, es decir no existe en el país producciones teóricas acerca del tema, teniendo escasa literatura nacional para el desarrollo de la presente investigación, por ello esto servirá para aportar conocimiento en cuanto el tema por ser realmente e innovadora.

Metodológico: Los resultados que se obtuvo de la investigación servirán como base legal para corregir y mejorar los vacíos legales en el Código Civil y establecer un reglamento de acuerdo al tiempo y espacio del Reniec, con el objetivo de mejorar la calidad de vida de los peruanos implementando un respeto y reconocimiento a su persona con un nombre de calidad y de esta manera disminuir las tasas de discriminación y bullying infantil como el laboral. Además servirá tanto para los ciudadanos como para el sistema judicial quienes ya no se verán afectados por la carga de trámites engorrosos que implica la modificación, supresión o adición del nombre. Las nuevas estrategias y políticas a tomar en cuenta a partir de este estudio mejorarán la situación actual que abarca la definición del problema propuesto. Además, es importante que se tome como antecedente a este estudio para nuevas investigaciones de cualquier índole; a fin de que coadyuven a solucionar el vacío legal de nuestra normatividad vigente.

\section{MATERIALES Y MÉTODOS}

Se realizó un análisis a las sentencias judiciales referentes al cambio, supresión o adición de nombre que fueron emitidas por los juzgados civiles o mixtos de Lima Metropolitana en el Perú durante los años 2010 al 2013, y completamos el estudio con el análisis de las informaciones proporcionada por el Reniec, todo ello basado en el nombre (específicamente el estudio se enfocó en los prenombres). Los métodos empleados son: No experimental porque no se manipuló variables ya que observamos los hechos tal y como se están dando en la realidad. Ex - Post facto ya que se estudio los hechos ya ocurridos. Transversal porque la recolección de datos se realizó en un solo momento y tiempo único. Se usó como herramienta de trabajo la base de datos proporcionada por la Corte Superior de Lima. La población es de 2772 sentencias con sus respectivos números de expedientes. Con ello se ingresó a la página web del Poder Judicial y evaluó los expedientes que corresponden con nuestra investigación y encontramos 70 expedientes referentes al cambio, supresión o adición de

Corte Superior de Justicia de Lima Norte

Fundada Infundada Improcedente

$78,26 \%$

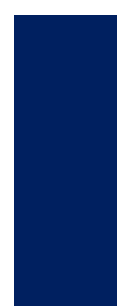

$17,39 \%$

Fundada Infundada

Improcedente

Figura 1. Corte Superior de Justicia de Lima Norte

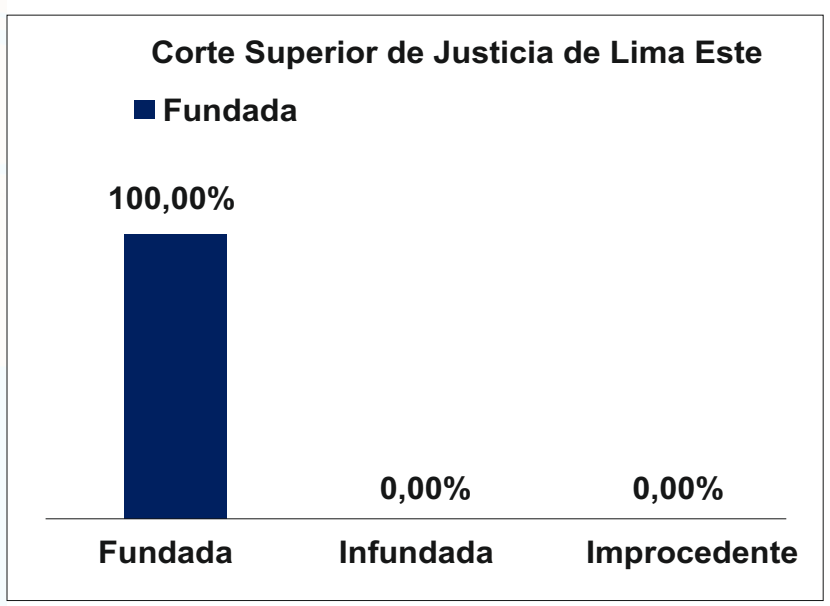

Figura 2. Corte Superior de Justicia de Lima Este

Corte Superior de Justicia de Lima Sur

Fundada $\quad$ Infundada $\square$ Improcedente

$58,33 \%$

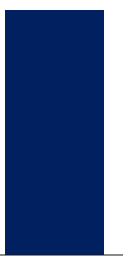

$41,67 \%$

Fundada

$0,00 \%$

Improcedente

Figura 3. Corte Superior de Justicia de Lima Sur 
Corte Superior de Justicia del Callao

- Fundada $\square$ Infundada $\square$ Improcedente

$80,00 \%$

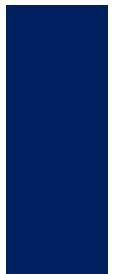

$10,00 \%$

$10,00 \%$

Fundada

Infundada

Improcedente

Figura 4. Corte Superior de Justicia del Callao

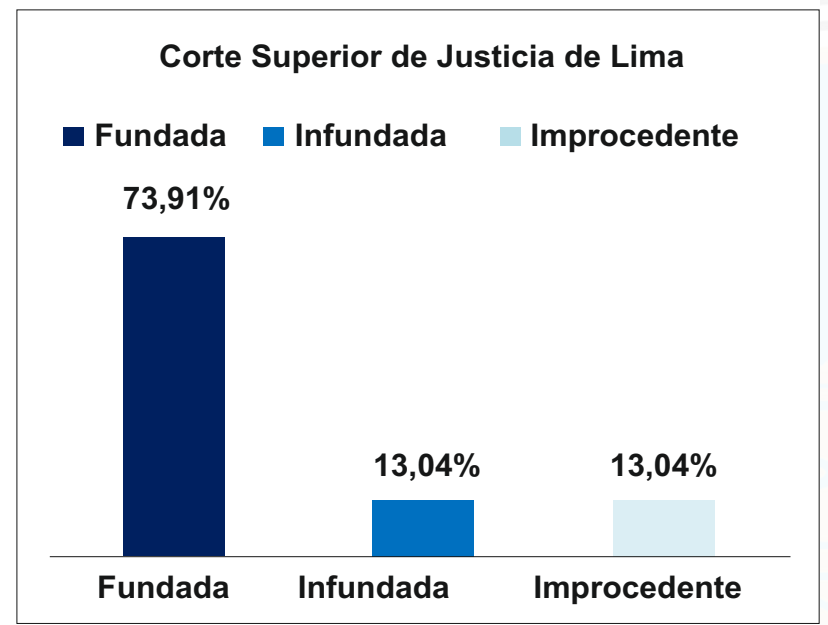

Figura 5. Corte Superior de Justicia de Lima

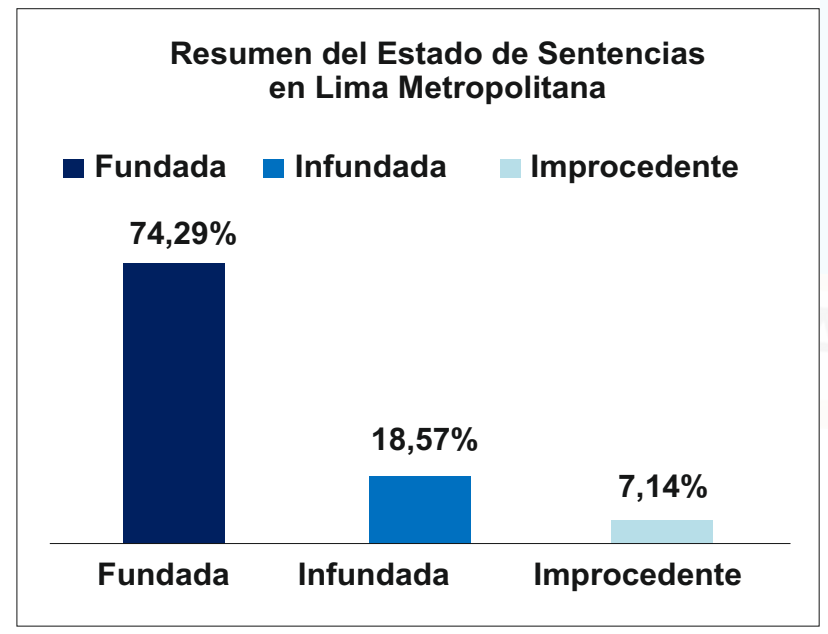

Figura 6. Resumen del Estado de Sentencias en Lima Metropolitana 